\title{
Hemodynamic study on upper extremity: simulation on straight reverse saphenous vein graft
}

\begin{abstract}
Artery reconstruction in upper extremities is rare performed compare to the incidence of reconstruction in lower extremities. In many cases, primary vascular repair was performed, whenever, otherwise, the interposition vein graft or venous bypass grafting were used in order to alleviate vascular occlusion. However, after grafting technique are applied, one or more of the digital arteries are blocked or severely narrowed because of mismatch of end-toside or end-to-end reverse saphenous vein graft. The objective of this study was to understand the end-to-end blood flow influence on reverse saphenous vein graft with small diameter. The finite volume method was employed to model the 3-D blood flow pattern to determine the velocity, pressure gradient, flow, wall shear stress, flow resistance and longitudinal impedance (ZL). We expected that reverse saphenous vein graft behave hydraulically like provide straight graft. Furthermore longitudinal impedance modulus (ZL) is expected to be inverse proportional to small diameter.
\end{abstract}

Keyword: Finite volume method; Upper extremity; Vein graft; Thrombosis; Longitudinal impedance 